\title{
Ermakov-Painlevé II Reduction in Cold Plasma Physics. Application of a Bäcklund Transformation
}

\author{
Colin Rogers ${ }^{\dagger}$ and Peter A. Clarkson ${ }^{\ddagger}$ \\ ${ }^{\dagger}$ School of Mathematics, The University of New South Wales, \\ Sydney, NSW2052, Australia \\ c.rogers@unsw.edu.au \\ ${ }^{\ddagger}$ School of Mathematics, Statistics \& Actuarial Science, \\ University of Kent, Canterbury, CT2 7FS, UK \\ P.A.Clarkson@kent.ac.uk
}

December 8, 2017

\begin{abstract}
A class of symmetry transformations of a type originally introduced in a nonlinear optics context is used here to isolate an integrable Ermakov-Painlevé II reduction of a resonant NLS equation which encapsulates a nonlinear system in cold plasma physics descriptive of the uniaxial propagation of magneto-acoustic waves. A Bäcklund transformation is employed in the iterative generation of novel classes of solutions to the cold plasma system which involve either Yablonski-Vorob'ev polynomials or classical Airy functions.
\end{abstract}

\section{Introduction}

The extensive connections between plasma physics, magnetohydrodynamics and canonical nonlinear integrable equations of modern soliton theory are well-documented. These links originated with pioneering work by Washimi and Taniuti [1] who employed reductive perturbation techniques to derive the Korteweg-de Vries (KdV) equation in the analysis of the propagation of ion-acoustic waves in a plasma. In contemporary work, Berezin and Karpman [2,3] independently derived the $\mathrm{KdV}$ equation in studies, both numerical and analytic, of large amplitude disturbances in plasmas and other dispersive media. Reviews of the role of the KdV equation in plasma physics and of the application of the reduction perturbation method to the study of hydrodynamic waves in cold plasma have been presented in [4] and [5] respectively. The integrable mkdV equation, related to the $\mathrm{KdV}$ equation by a Miura transformation, likewise arises in the analysis of the propagation of nonlinear Alfvén waves in cold collisionless plasma [6]. In addition, the nonlinear Schrödinger equation has an extensive literature concerned with its derivation in plasma physics in a variety of contexts. Thus, in particular, its occurrence in the analysis of the propagation of Langmuir waves in plasma has been described in [7-9]. The text [10] provides an account of perturbation methods adduced in the derivation of the NLS equation in plasma physics, notably with regard to incorporation of Landau damping phenomena.

An integrable Heisenberg spin equation associated with the NLS equation has been derived in spatial gasdynamics and magneto-hydrostatics in $[11,12]$ via geometric methods originally applied in a magnetohydrodynamic context in [13]. This purely geometric formalism as opposed to a reductive perturbation approach may be shown to lead to a classical integrable da Rios system reduction in complex-lamellar magnetohydrodynamics [14]. It has also been applied not only to construct the auto-Bäcklund transformation for the auto-Bäcklund transformation for the NLS 
equation in a purely geometric manner $[15,16]$ but also to obtain a novel integrable PohlmeyerLund-Regge reduction in magnetohydrodynamics $[17,18]$. The combined action of Bäcklund and reciprocal-type transformations has been used, via an integrable sinh-Gordon reduction, to construct periodic solutions of breather-type in super-Alfvénic magnetogasdynamics in [19]. Invariance under multi-parameter reciprocal transformations has been established in two-dimensional orthogonal magnetogasdynamics in [20].

In $[21,22]$ elliptic vortex solutions in magnetogasdynamics have been obtained via symmetry reduction to an integrable Ermakov-Ray-Reid system with underlying Hamiltonian structure. Ermakov-Ray-Reid systems have diverse physical applications, in particular, in nonlinear optics and rotating shallow water theory (see e.g. [23-25]). Moreover, in [26], a 2+1-dimensional nonisothermal magnetogasdynamic version of a gas cloud system with origin in work of Dyson [27] was shown to admit symmetry reduction to an eight dimensional nonlinear dynamical subsystem with underlying Hamiltonian-Ermakov structure. A Lax pair representation was constructed for this integrable subsystem.

Here, a class of wave packet representations of a type originally introduced in a nonlinear optics context in [28] is used to isolate a Ermakov-Painlevé II symmetry reduction of a resonant NLS equation which encapsulates a $1+1$-dimensional cold plasma physics system. The latter describes the propagation of uniaxial long magnetoacoustic waves in a cold collisionless plasma subject to a transverse magnetic field. Iterative application of a Bäcklund transformation is used to generate novel classes of exact solutions of the cold plasma system in terms of either Yablonski-Vorob'ev polynomials or classical Airy functions.

\section{The Two-Component Cold Plasma System. A Resonant NLS Encapsulation}

The dynamics of two-component cold collisionless plasma in the presence of an external magnetic field $\mathbf{B}$ is governed by the nonlinear system of equations $[29,30]$.

$$
\begin{aligned}
& m_{i}\left[\partial / \partial t+\mathbf{v}_{i} \nabla\right] \mathbf{v}_{i}=e\left[\mathbf{E}+\left(\mathbf{v}_{i} \times \mathbf{B}\right)\right] \\
& m_{e}\left[\partial / \partial t+\mathbf{v}_{e} \nabla\right] \mathbf{v}_{e}=-e\left[\mathbf{E}+\left(\mathbf{v}_{e} \times \mathbf{B}\right)\right] \\
& \frac{\partial n_{i}}{\partial t}+\nabla \bullet\left(n_{i} \mathbf{v}_{i}\right)=0 \\
& \frac{\partial n_{e}}{\partial t}+\nabla \cdot\left(n_{e} \mathbf{v}_{e}\right)=0 \\
& \operatorname{curl} \mathbf{B}=\mathrm{e} \mu_{0}\left(\mathrm{n}_{\mathrm{i}} \mathbf{v}_{\mathrm{i}}-\mathrm{n}_{\mathrm{e}} \mathbf{v}_{\mathrm{e}}\right) \\
& \frac{\partial \mathbf{B}}{\partial t}=-\operatorname{curl} \mathbf{E} \\
& \nabla \cdot \mathbf{B}=0
\end{aligned}
$$

where $m_{i}, m_{e}, \mathbf{v}_{i}, \mathbf{v}_{e}, n_{i}, n_{e}$ denote, in turn, masses, velocities and concentrations of ions and electrons respectively. $\mathbf{E}$ is the electric field, $\mathbf{B}$ is the magnetic field, $e$ is the electric charge and $\mu_{0}$ is the magnetic permeability. If the oscillation frequency is much smaller than the ion Langmuir frequency, then plasma quasi-neutrality is implied, that is, $n_{i} \approx n_{e}=n$.

On introduction of the mass density $\rho$ and velocity $\mathbf{u}$ according to

$$
\rho=\left(m_{i}+m_{e}\right) n, \quad \mathbf{u}=\frac{m_{i} \mathbf{v}_{i}+m_{e} \mathbf{v}_{e}}{m_{i}+m_{e}},
$$

then, on elimination of the electric field $\mathbf{E}$, if $m_{e} / m_{i} \ll 1$, in the case of uni-axial plasma propagation with transverse magnetic field $\mathbf{B}$, so that

$$
\mathbf{u}=u(x, t) \mathbf{i}, \quad \mathbf{B}=B(x, t) \mathbf{k},
$$


the system (2.1) may be reduced to the form [31]

$$
\begin{aligned}
& \frac{\partial \rho}{\partial t}+\frac{\partial}{\partial x}(\rho u)=0 \\
& \rho\left(\frac{\partial u}{\partial t}+u \frac{\partial u}{\partial x}\right)+B \frac{\partial B}{\partial x}=0 \\
& \frac{\partial}{\partial t}\left[B-\frac{\partial}{\partial x}\left(\frac{1}{\rho} \frac{\partial B}{\partial x}\right)\right]+\frac{\partial}{\partial x}\left\{u\left[B-\frac{\partial}{\partial x}\left(\frac{1}{\rho} \frac{\partial B}{\partial x}\right)\right]\right\}=0 .
\end{aligned}
$$

Introduction of the Lagrangian variable $X(x, t)$ via the continuity equation (2.3a) according to

$$
d X=\rho d x-\rho u d t
$$

into $(2.3 \mathrm{c})$ shows that

$$
B-\frac{\partial}{\partial x}\left(\frac{1}{\rho} \frac{\partial B}{\partial x}\right)=M(X) \rho .
$$

The system consisting of $(2.3 \mathrm{a}),(2.3 \mathrm{~b})$ augmented by $(2.4)$ with $M(X)=1$ was set down in a nonlinear dispersive wave context by Whitham [32]. In the present context it describes the propagation of $1+1$-dimensional nonlinear magnetoacoustic waves in a cold plasma subject to a transverse magnetic field.

Here, we consider a shallow water type approximation to the Whitham system consisting of the continuity and momentum equations (2.3a), (2.3b) together with

$$
B-\frac{\partial}{\partial x}\left(\frac{1}{\rho} \frac{\partial B}{\partial x}\right)=\rho .
$$

Thus, on re-scaling the space and time variables via $x \rightarrow \beta x, t \rightarrow \beta t$ and expansion of the expression for the magnetic field $B$ in the parameter $\beta^{2}$ according to [31]

$$
B=\rho+\beta^{2} \mathcal{B}\left(\rho, \rho_{x}, \rho_{x x}, \ldots\right)+\mathcal{O}\left(\beta^{4}\right),
$$

insertion into (2.5) yields

$$
\mathcal{B}=\frac{\partial}{\partial x}\left(\frac{1}{\rho} \frac{\partial \rho}{\partial x}\right)
$$

Thus, to $\mathcal{O}\left(\beta^{2}\right)$, the following nonlinear system results [31]:

$$
\begin{aligned}
& \frac{\partial \rho}{\partial t}+\frac{\partial}{\partial x}(\rho u)=0 \\
& \frac{\partial u}{\partial t}+u \frac{\partial u}{\partial x}+\beta^{2} \frac{\partial}{\partial x}\left[\frac{1}{\rho} \frac{\partial^{2} \rho}{\partial x^{2}}-\frac{1}{2}\left(\frac{1}{\rho} \frac{\partial \rho}{\partial x}\right)^{2}+\frac{\rho}{\beta^{2}}\right] .
\end{aligned}
$$

This describes the uniaxial propagation of long magneto-acoustic waves in a cold plasma with velocity magnitude $u$ and magnetic field given by (2.2) together with (2.6)-(2.7).

On use of the one-dimensional version of a relation for the de Broglie-Bohm potential, namely

$$
\frac{\nabla^{2} \rho^{1 / 2}}{\rho^{1 / 2}}=\frac{\nabla^{2} \rho}{\rho}-\frac{1}{4}\left(\frac{\nabla \rho}{\rho}\right)^{2},
$$

it seen that the cold plasma momentum equation in (2.8) becomes

$$
\frac{\partial u}{\partial t}+u \frac{\partial u}{\partial x}+\beta^{2} \frac{\partial}{\partial x}\left[2 \frac{\left(\rho^{1 / 2}\right)_{x x}}{\rho^{1 / 2}}+\frac{\rho}{\beta^{2}}\right]=0 .
$$


Introduction of the velocity potential $S$ according to $u=-2 \partial S / \partial x$ into the continuity equation $(2.8)_{1}$ and momentum equation (2.9) in turn, gives

$$
\frac{\partial \rho}{\partial t}-2 \frac{\partial}{\partial x}\left(\rho \frac{\partial S}{\partial x}\right)=0
$$

together with the Bernoulli integral

$$
-\frac{\partial S}{\partial t}+\left(\frac{\partial S}{\partial x}\right)^{2}+\frac{\rho}{2}+\frac{\beta^{2}}{\rho^{1 / 2}} \frac{\partial^{2}}{\partial x^{2}}\left(\rho^{1 / 2}\right)=T(t) .
$$

In the latter, $T(t)$ may be absorbed into the potential $S$ and so may be set zero without loss of generality.

If the classical Madelung transformation is now introduced according to [33]

$$
\Psi=\rho^{1 / 2} e^{-i S}
$$

then it is seen that the cold plasma system (2.8) may be encapsulated in the resonant NLS equation $[34,35]$

$$
i \frac{\partial \Psi}{\partial t}+\frac{\partial^{2} \Psi}{\partial x^{2}}-\frac{1}{2}|\Psi|^{2} \Psi=\left(1+\beta^{2}\right) \frac{\Psi}{|\Psi|} \frac{\partial^{2}|\Psi|}{\partial x^{2}},
$$

which incorporates a de Broglie-Bohm potential term $\frac{1}{|\Psi|} \frac{\partial^{2}}{\partial x^{2}}|\Psi|$. It is recalled that, if $s<1$ then the canonical $1+1$-dimensional resonant NLS equation

$$
i \frac{\partial \Psi}{\partial t}+\frac{\partial^{2} \Psi}{\partial x^{2}}+\nu|\Psi|^{2} \Psi=\frac{s}{|\Psi|} \frac{\partial^{2}|\Psi|}{\partial x^{2}},
$$

can be transformed to the standard integrable cubic NLS equation with the de Broglie-Bohm term removed [34-36]. However, if as in the present cold plasma case $s=1+\beta^{2}>1$ this reduction to remove the de Broglie Bohm term is not available. Then, as shown in [31] the resonant NLS equation (2.10) which encapsulates the cold plasma system is equivalent to a canonical two-component system contained in the AKNS hierarchy of solitonic systems amenable to the inverse scattering transform $[37,38]$. Bäcklund-Darboux transformations and concomitant nonlinear superposition principles for the resonant NLS equation and hence for the cold plasma system which it encapsulates have been constructed in [31]. This resonant NLS equation importantly, unlike its standard integrable NLS counterpart can admit solitonic fusion or fission phenomena [35]. Here, wave packet solutions of the resonant NLS equation (2.10) are generated via a symmetry reduction to a prototype integrable Ermakov-Painlevé II equation [39,40]. The iterated application of a Bäcklund transformation then allows the construction of novel classes of exact solutions to the cold plasma system in terms of either Yablonski-Vorob'ev polynomials or classical Airy functions.

\section{Ermakov-Painlevé II Symmetry Reduction}

Here, symmetry reduction of the resonant NLS equation (2.10) is sought under the wave packet ansatz

$$
\begin{aligned}
\Psi=\rho^{1 / 2} e^{-i S} & =[\phi(\xi)+i \psi(\xi)] e^{i \eta}, \\
\xi & =\alpha t+\beta t^{2}+\gamma x, \\
\eta & =\gamma t^{3}+\delta t^{2}+\varepsilon \gamma t x+\zeta t+\lambda x,
\end{aligned}
$$


with $\alpha, \beta, \gamma, \delta, \varepsilon, \zeta$ and $\lambda$ arbitrary constants. Insertion of the latter into (2.10) produces the coupled nonlinear system

$$
\begin{aligned}
& \gamma^{2} \frac{d^{2} \phi}{d \xi^{2}}-\frac{d \psi}{d \xi}\left[2\left(\beta+\varepsilon \gamma^{2}\right) t+\alpha+2 \lambda \gamma\right]-\Delta \phi=0 \\
& \gamma^{2} \frac{d^{2} \psi}{d \xi^{2}}+\frac{d \phi}{d \xi}\left[2\left(\beta+\varepsilon \gamma^{2}\right) t+\alpha+2 \lambda \gamma\right]-\Delta \psi=0
\end{aligned}
$$

where

$$
\Delta=3 \gamma t^{2}+2 \delta t+\varepsilon \gamma x+\zeta+(\varepsilon \gamma t+\lambda)^{2}+\frac{1}{2}|\Psi|^{2}+\frac{s \gamma^{2}}{|\Psi|} \frac{d^{2}|\Psi|}{d \xi^{2}} .
$$

and $s=1+\beta^{2}>1$.

The relations (3.2) combine to show that

$$
\gamma^{2}\left(\psi \frac{d^{2} \phi}{d \xi^{2}}-\phi \frac{d^{2} \psi}{d \xi^{2}}\right)-\left(\phi \frac{d \phi}{d \xi}+\psi \frac{d \psi}{d \xi}\right)\left[2\left(\beta+\varepsilon \gamma^{2}\right) t+\alpha+2 \lambda \gamma\right]=0
$$

whence, it is required that

$$
\beta+\varepsilon \gamma^{2}=0
$$

in which case, (3.4) admits the integral

$$
\gamma^{2}\left(\psi \frac{d \phi}{d \xi}-\phi \frac{d \psi}{d \xi}\right)-\frac{1}{2}(\alpha+2 \lambda \gamma)|\Psi|^{2}=\mathcal{I}
$$

where $\mathcal{I}$ is an arbitrary constant.

It is seen that (3.3) may be written as, if $\beta \neq 0$

$$
\begin{aligned}
\Delta= & \gamma \beta^{-1}\left(\varepsilon^{2} \gamma+3\right)(\xi-\alpha t-\gamma x)+2(\delta+\varepsilon \gamma \lambda) t+\varepsilon \gamma x+\zeta+\lambda^{2} \\
& \quad+\frac{1}{2}|\Psi|^{2}+\frac{s \gamma^{2}}{|\Psi|} \frac{d^{2}|\Psi|}{d \xi^{2}} \\
= & \varepsilon \xi+\zeta+\lambda^{2}+\frac{1}{2}|\Psi|^{2}+\frac{s \gamma^{2}}{|\Psi|} \frac{d^{2}|\Psi|}{d \xi^{2}}
\end{aligned}
$$

on setting

$$
\alpha \varepsilon=2(\delta+\varepsilon \gamma \lambda), \quad \beta \varepsilon=\gamma\left(3+\varepsilon^{2} \gamma\right) .
$$

Moreover, (3.2) show that

$$
\gamma^{2}\left(\phi \frac{d^{2} \phi}{d \xi^{2}}+\psi \frac{d^{2} \psi}{d \xi^{2}}\right)+\left(\psi \frac{d \phi}{d \xi}-\phi \frac{d \psi}{d \xi}\right)(\alpha+2 \lambda \gamma)-\Delta|\Psi|^{2}=0
$$

whence, on use of the relations

$$
\left[\phi \frac{d^{2} \phi}{d \xi^{2}}+\psi \frac{d^{2} \psi}{d \xi^{2}}+\left(\frac{d \phi}{d \xi}\right)^{2}+\left(\frac{d \psi}{d \xi}\right)^{2}\right]\left(\phi^{2}+\psi^{2}\right)-\left(\phi \frac{d \phi}{d \xi}+\psi \frac{d \psi}{d \xi}\right)^{2}=\frac{d^{2}|\Psi|}{d \xi^{2}}|\Psi|^{3},
$$

and

$$
\left(\phi^{2}+\psi^{2}\right)\left[\left(\frac{d \phi}{d \xi}\right)^{2}+\left(\frac{d \psi}{d \xi}\right)^{2}\right]-\left(\psi \frac{d \phi}{d \xi}-\phi \frac{d \psi}{d \xi}\right)^{2}=\left(\phi \frac{d \phi}{d \xi}+\psi \frac{d \psi}{d \xi}\right)^{2}
$$

it is seen that

$$
\gamma^{2}\left[\frac{d^{2}|\Psi|}{d \xi^{2}}|\Psi|^{3}-\left(\psi \frac{d \phi}{d \xi}-\phi \frac{d \psi}{d \xi}\right)^{2}\right]+(\alpha+2 \lambda \gamma)\left(\psi \frac{d \phi}{d \xi}-\phi \frac{d \psi}{d \xi}\right)|\Psi|^{2}-\Delta|\Psi|^{4}=0
$$


The latter, by virtue of the integral of motion (3.5) and the expression (3.6) for $\Delta$ now produces a hybrid Ermakov-Painlevé equation in the amplitude $|\Psi|$ namely

$$
|\Psi|_{\xi \xi}+\left(c_{1}+c_{2} \xi\right)|\Psi|+c_{3}|\Psi|^{3}=\frac{\mathcal{I}^{2}}{(1-s) \gamma^{4}|\Psi|^{3}},
$$

where the constants $c_{1}, c_{2}$ and $c_{3}$ are given by

$$
c_{1}=\frac{1}{(1-s) \gamma^{4}}\left[\left(\alpha-\frac{\delta}{\varepsilon}\right)^{2}-\gamma^{2}\left(\zeta+\lambda^{2}\right)\right], \quad c_{2}=\frac{\varepsilon}{(s-1) \gamma^{2}}, \quad c_{3}=\frac{1}{2(s-1) \gamma^{2}} .
$$

The type of nonlinear equation (3.7) has been previously shown to arise in connection with a pair of three-ion cases in the classical Nernst-Planck electrodiffusion system [40]. Here, $|\Psi|=\rho^{1 / 2}$ and in terms of the density $\rho$ it is seen that (3.7) becomes

$$
\frac{d^{2} \rho}{d \xi^{2}}-\frac{1}{2 \rho}\left(\frac{d \rho}{d \xi}\right)^{2}+2\left[c_{1}+c_{2} \xi\right] \rho+2 c_{3} \rho^{2}+\frac{2 \mathcal{I}^{2}}{(s-1) \gamma^{4} \rho}=0
$$

which with the admissible specialisations,

$$
c_{1}=0, \quad c_{2}=\frac{1}{2}, \quad c_{3}=1, \quad \frac{4 \mathcal{I}^{2}}{(s-1) \gamma^{4}}=\left(\alpha+\frac{1}{2}\right)^{2}
$$

becomes the canonical integrable Painlevé XXXIV $\left(\mathrm{P}_{34}\right)$ equation in $\rho>0$, namely

$$
\frac{d^{2} \rho}{d \xi^{2}}=\frac{1}{2 \rho}\left(\frac{d \rho}{d \xi}\right)^{2}+2 \rho^{2}-\xi \rho-\frac{\left(\alpha+\frac{1}{2}\right)^{2}}{2 \rho},
$$

cf. Gromak [41]. It is emphasised that the relation (3.8) involving the Painlevé parameter $\alpha$, necessarily requires that $s>1$ as indeed is the case for the present cold plasma system incapsulated in a nonlinear resonant NLS equation. The regions of positivity of classes of solutions of $\mathrm{P}_{34}(3.9)$ as generated by the iterated action of a Bäcklund transformation have been investigated in the context of boundary value problems in two-ion electrodiffusion in [42]. Therein, the ion concentrations $c_{ \pm}$, which are necessarily positive, are governed by solutions of $\mathrm{P}_{34}(3.9)$.

With a positive solution $\rho=|\Psi|^{2}=\phi^{2}+\psi^{2}$ of $\mathrm{P}_{34}$ (3.9) to hand, the ratio $\psi / \phi$ is determined by the integral of motion (3.5). The latter yields

$$
-\gamma^{2} \frac{d}{d \xi}\left[\tan ^{-1}\left(\frac{\psi}{\phi}\right)\right]-\frac{1}{2}(\alpha+2 \lambda \gamma)=\frac{\mathcal{I}}{|\Psi|^{2}}
$$

so that, on integration,

$$
\gamma^{2} \tan ^{-1}\left(\frac{\psi}{\phi}\right)+\frac{1}{2}(\alpha+2 \lambda \gamma) \xi+\mathcal{I} \int \frac{d \xi}{\rho}=K
$$

where $K$ is an arbitrary constant. Thus, with $\Lambda=\psi / \phi$, it is seen that the $\phi, \psi$ in the original wave packet represenation (3.1) are given by the relations

$$
\phi= \pm \frac{|\Psi|}{\sqrt{1+\Lambda^{2}}}, \quad \psi= \pm \frac{\Lambda|\Psi|}{\sqrt{1+\Lambda^{2}}}
$$

The velocity magnitude in the cold plasma system (2.8) is given by $u=\Phi_{x}$ where $\Phi$ is the potential given by

$$
\Phi=2 \tan ^{-1}\left(\frac{\psi+\phi \tan \eta}{\phi-\psi \tan \eta}\right)=2\left[\tan ^{-1}\left(\frac{\psi}{\phi}\right)+\eta\right]
$$


on use of the identity

$$
\tan ^{-1}\left(\frac{x+y}{1-x y}\right) \equiv \tan ^{-1} x+\tan ^{-1} y
$$

Thus,

$$
\begin{aligned}
u & =2\left[\gamma \frac{\partial}{\partial \xi}+(\varepsilon \gamma t+\lambda) \frac{\partial}{\partial \eta}\right]\left[-\frac{1}{2 \gamma^{2}}(\alpha+2 \lambda \gamma) \xi-\frac{\mathcal{I}}{\gamma^{2}} \int \frac{d \xi}{\rho(\xi)}+\eta\right] \\
& =-\frac{2 \mathcal{I}}{\gamma \rho(\xi)}+2 \varepsilon \gamma t-\frac{\alpha}{\gamma} .
\end{aligned}
$$

while the term $\mathcal{B}$ in the expansion (2.6) for the magnetic field $B$ is given by

$$
\mathcal{B}=\gamma^{2} \frac{d^{2}}{d \xi^{2}} \ln \rho(\xi)
$$

where the density $\rho(\xi)$ is governed by $\mathrm{P}_{34}(3.9)$.

In the sequel, a link between the Ermakov-Painlevé II equation for the density $\rho(\xi)$ and the Painlevé II $\left(\mathrm{P}_{\mathrm{II}}\right)$ equation

$$
\frac{d^{2} q}{d \xi^{2}}=2 q^{3}+\xi q+\alpha
$$

with $\alpha$ a parameter, via $\mathrm{P}_{34}(3.9)$ is exploited to construct novel classes of wave packet solutions of the cold plasma system in terms of Yablonski-Vorob'ev polynomials or classical Airy functions via the iterated application of the well-known Bäcklund transformation for $\mathrm{P}_{\text {II }}[43,44]$; see also $[45,46]$. These solutions may, in turn, be used as seed solutions in the application of the Bäcklund-Darboux transformations as established for the resonant NLS equation in [31].

\section{Iterative Action of a Bäcklund Transformation}

Bäcklund transformations have established applications in continuum mechanics and notably in modern soliton theory $[16,47]$. These have roots, in turn, in work of Loewner $[48,49]$ on model laws in gasdynamics and Seeger et al. [50] on crystal dislocations. Thus, Loewner applied novel matrix Bäcklund transformations to construct multi-parameter gas laws for which the classical hodograph system may be reduced to appropriate canonical forms in subsonic, transonic and supersonic flow régimes. Seeger et al. [50], on the other hand, in the context of Frenkel and Kontorova's dislocation theory isolated the nonlinear interaction of what they termed 'eigenmotions' via Bianchi's classical permutability theorem as derived via the auto-Bäcklund transformation for the sine Gordon equation of pseudo-spherical surface thoery. In particular, the interaction process of what, in soliton theory came to be called a breather and kink was described analytically by means of this nonlinear superposition principle. Importantly, Lamb [51] later exploited this permutability theorm to predict experimentally observed decomposition of ultrashort optical pulses in a resonant medium.

The areas of application of Bäcklund transformations in nonlinear continuum mechanics and modern soliton theory were brought together in $[52,53]$ where it was established that a re-interpretation and extension of the class of matrix infinitesimal Bäcklund transformations introduced by Loewner in a gasdynamics setting provide a linear representation for a master $2+1$-dimensional soliton system. Basic reductions of this system lead, in particular to novel integrable $2+1$-dimensional versions of the principal chiral fields model, Toda lattice system and, notably, of the classical sine Gordon equation [53-61]. It is remarked that the paper of Loewner [48] contains, 'mutatis mutandis' a linear representation for the 1+1-dimensional sine Gordon equation which is gauge equivalent to that later obtained in the celebrated AKNS system [37].

The preceding attests to the seminal role that Bäcklund transformations have played in modern soliton theory. Likewise the classical Painlevé equations and the Bäcklund transformations they 
admit arise naturally in the study of integrable solitonic systems (see e.g. [41, 45, 46, 62-64] and work cited therein). Here, our concern is with the application of a Bäcklund transformation for $\mathrm{P}_{\mathrm{II}}$ (3.10) to the present symmetry reduction of the cold plasma system encapsulated in the resonant NLS equation (2.10).

It turns out that, remarkably, all known exact solutions of $\mathrm{P}_{\mathrm{II}}(3.10)$ and hence also of $\mathrm{P}_{34}(3.9)$ may be generated via iteration of a Bäcklund transformation of (3.10) due to Gambier [43] and later Lukashevich [44].

The well-known relationship between solutions of $\mathrm{P}_{\mathrm{II}}$ (3.10) and solutions of $\mathrm{P}_{34}$ (3.9) is derived via the Hamiltonian system

$$
\frac{d q}{d \xi}=\frac{\partial \mathcal{H}_{\mathrm{II}}}{\partial \rho}, \quad \frac{d \rho}{d \xi}=-\frac{\partial \mathcal{H}_{\mathrm{II}}}{\partial q},
$$

where the Hamiltonian $\mathcal{H}_{\mathrm{II}}(\rho, q, \xi ; \alpha)$ is given by

$$
\mathcal{H}_{\mathrm{II}}(\rho, q, \xi ; \alpha)=\frac{1}{2} \rho^{2}-\left(q^{2}+\frac{1}{2} \xi\right) \rho-\left(\alpha+\frac{1}{2}\right) q,
$$

which yield the nonlinear system

$$
\frac{d q}{d \xi}=\rho-q^{2}-\frac{1}{2} \xi, \quad \frac{d \rho}{d \xi}=2 q \rho+\alpha+\frac{1}{2},
$$

see $[65,66]$. Elimination of $\rho$ and $q$ successively in (4.1) yields $\mathrm{P}_{\mathrm{II}}(3.10)$ and $\mathrm{P}_{34}(3.9)$.

If $q_{\alpha}(\xi)=q(\xi ; \alpha)$ is a solution of $\mathrm{P}_{\mathrm{II}}(3.10)$ with parameter $\alpha$, then

$$
\begin{aligned}
& q_{\alpha+1}(\xi)=-q_{\alpha}(\xi)-\frac{2 \alpha+1}{2 q_{\alpha}^{\prime}(\xi)+2 q_{\alpha}^{2}(\xi)+\xi}, \\
& q_{\alpha-1}(\xi)=-q_{\alpha}(\xi)-\frac{2 \alpha-1}{2 q_{\alpha}^{\prime}(\xi)-2 q_{\alpha}^{2}(\xi)+\xi},
\end{aligned}
$$

with $^{\prime} \equiv d / d \xi$, which are the Bäcklund transformations of $\mathrm{P}_{\mathrm{II}}(3.10)[43,44]$. If $\rho_{\alpha}(\xi)=\rho(\xi ; \alpha)$ is a solution of $\mathrm{P}_{34}$ (3.9) with parameter $\alpha$, then from (4.1) we have

$$
\rho_{\alpha}=\frac{d q_{\alpha}}{d \xi}+q_{\alpha}^{2}+\frac{1}{2} \xi, \quad q_{\alpha}=\frac{1}{2 \rho_{\alpha}}\left(\frac{d \rho_{\alpha}}{d \xi}-\alpha-\frac{1}{2}\right) .
$$

Consequently, from (4.2) and (4.3) we obtain the Bäcklund transformations of $\mathrm{P}_{34}(3.9)$ given by

$$
\begin{aligned}
& \rho_{\alpha+1}=z-\rho_{\alpha}+\frac{1}{2 \rho_{\alpha}^{2}}\left(\frac{d \rho_{\alpha}}{d \xi}+\alpha+\frac{1}{2}\right)^{2} \\
& \rho_{\alpha-1}=z-\rho_{\alpha}+\frac{1}{2 \rho_{\alpha}^{2}}\left(\frac{d \rho_{\alpha}}{d \xi}-\alpha-\frac{1}{2}\right)^{2}
\end{aligned}
$$

see also $[66,67]$

In the present plasma physics context, in particular, this produces an extensive class of exact rational solutions for the density $\rho$ governed by $\mathrm{P}_{34}(3.9)$ given by

$$
\rho=\rho_{+}(\xi ; n)=\frac{Q_{n+1}(\xi) Q_{n-1}(\xi)}{2 Q_{n}^{2}(\xi)}, \quad n \in \mathbb{N},
$$

with $\rho_{+}(\xi ; 0)=\frac{1}{2} \xi$, corresponding to the parameters $\alpha=n$, where the $Q_{n}(\xi)$ are the YablonskiiVorob'ev polynomials determined by the quadratic recurrence relations $[68,69]$

$$
Q_{n+1} Q_{n-1}=\xi Q_{n}^{2}+4\left[\left(\frac{d Q_{n}}{d \xi}\right)^{2}-Q_{n} \frac{d^{2} Q_{n}}{d \xi^{2}}\right]
$$


with $Q_{-1}(\xi)=Q_{0}(\xi)=1$. The rational solutions of $\mathrm{P}_{34}$ (3.9) can also be expressed in terms of determinants. Let $p_{k}(\xi)$ be the polynomial defined by

$$
\sum_{k=0}^{\infty} p_{k}(\xi) \lambda^{k}=\exp \left(\xi \lambda-\frac{4}{3} \lambda^{3}\right),
$$

and $\tau_{n}(\xi)$ be the Wronskian

$$
\tau_{n}(\xi)=\mathcal{W}\left(p_{1}(\xi), p_{3}(\xi), \ldots, p_{2 n-1}(\xi)\right) \equiv\left|\begin{array}{cccc}
p_{1}(\xi) & p_{3}(\xi) & \cdots & p_{2 n-1}(\xi) \\
p_{1}^{\prime}(\xi) & p_{3}^{\prime}(\xi) & \cdots & p_{2 n-1}^{\prime}(\xi) \\
\vdots & \vdots & \ddots & \vdots \\
p_{1}^{(n-1)}(\xi) & p_{3}^{(n-1)}(\xi) & \cdots & p_{2 n-1}^{(n-1)}(\xi)
\end{array}\right|
$$

for $n \geq 1$. Then the rational solution

$$
\rho_{+}(\xi ; n)=\frac{1}{2} \xi-2 \frac{d^{2}}{d \xi^{2}} \ln \tau_{n}(\xi)
$$

satisfies $\mathrm{P}_{34}(3.9)$ with $\alpha=n$.

Iterated action of the Bäcklund transformations (4.4) for $\mathrm{P}_{34}(3.9)$ on the seed Airy-type solution with parameter $\alpha=\frac{1}{2}$ generates a class of exact solutions of $\mathrm{P}_{34}(3.9)$ given by

$$
\rho\left(\xi ; n-\frac{1}{2}\right)=\frac{u_{n-1}(\xi) u_{n+1}(\xi)}{2 u_{n}^{2}(\xi)}, \quad n \in \mathbb{N},
$$

for the parameter $\alpha=n-\frac{1}{2}$ and where the sequence $\left.\left\{u_{n} \xi\right)\right\}$, for $n \geq 0$, is determined by the Toda recurrence relation

$$
u_{n+1} u_{n-1}=4\left[\left(\frac{d u_{n}}{d \xi}\right)^{2}-u_{n} \frac{d^{2} u_{n}}{d \xi^{2}}\right]
$$

with initial values $u_{0}(\xi)=1$ and $u_{1}(\xi)=\varphi(\xi)$, where $\varphi(\xi)$ is governed by the classical Airy equation

$$
\frac{d^{2} \varphi}{d \xi^{2}}+\frac{1}{2} \xi \varphi=0
$$

i.e.

$$
\varphi(\xi)=a \operatorname{Ai}(z)+b \operatorname{Bi}(z), \quad z=-2^{-1 / 3} \xi,
$$

with $\operatorname{Ai}(z)$ and $\operatorname{Bi}(z)$ the Airy functions and $a, b$ arbitrary parameters. The Airy-type solutions of $\mathrm{P}_{34}$ (3.9) can also be expressed in terms of determinants. Suppose that $\Phi_{n}(\xi)$ is the Hankel $n \times n$ determinant

$$
\Phi_{n}(\xi)=\left[\frac{d^{j+k}}{d \xi^{j+k}} \varphi(\xi)\right]_{j, k=0}^{n-1}, \quad n \geq 1,
$$

with $\varphi(\xi)$ given by $(4.11)$ and $\Phi_{0}(\xi)=1$, then for $n \geq 1$,

$$
p\left(\xi ; n-\frac{1}{2}\right)=-2 \frac{d^{2}}{d \xi^{2}} \ln \Phi_{n}(\xi),
$$

satisfies $\mathrm{P}_{34}(3.9)$ with $\alpha=n-\frac{1}{2}$, cf. [70].

In previous work in [42], solutions in terms of $\mathrm{P}_{34}$ in a two-ion electro-diffusion context have been used to investigate certain boundary value problems. There the positivity requirement on the solutions arises via such a constraint on the ion concentrations. Here, it arises due to the positivity contraint on the density. The rational solutions of $\mathrm{P}_{34}$ are not positive for all $\xi$. However, positivity 
may be established in certain regions $\xi>c$, a constant. Thus, the rational solutions of $\mathrm{P}_{34}$ are given by

$$
p[k]=\frac{1}{2} \xi-2 \frac{d^{2}}{d \xi^{2}} \ln P_{k-1}(\xi), \quad p[-1]=\frac{1}{2} \xi
$$

where the $P_{k}$ are the Yablonski-Vorob'ev polynomials, cf. [71,72]. If $\xi_{k-1}$ denotes the largest real zero of the polynomial $P_{k-1}$ for each $k \in \mathbb{N}$ then the constraint $\xi_{k-1}<c$ ensures positivity in the region $\xi>c$. The positivity property is illustrated graphically for $k=0,1,2$ in [42].

The Airy-type solutions of $\mathrm{P}_{\text {II }}$ admit the representation $[42,70]$

$$
Y\left[k-\frac{1}{2}\right]=\frac{d}{d \xi} \ln \frac{\psi_{k-2}}{\psi_{k-1}}-\Phi,
$$

wherein the sequence $\left\{\psi_{k}\right\}_{k \geq-1}$ is given by the recurrence relation

$$
\psi_{k+1} \psi_{k-1}=4\left[\left(\frac{d \psi_{k}}{d \xi}\right)^{2}-\psi_{k} \frac{d^{2} \psi_{k}}{d \xi^{2}}+\frac{1}{2}(k+1)\left(2 \Phi^{2}+\xi\right) \psi_{k}^{2}\right]
$$

together with the initial values $\psi_{-1}=1, \psi_{0}=1$. In the above $\Phi=(\ln \phi)^{\prime}$ where $\phi$ is, in general, given by (4.11). The corresponding Airy-type solutions of $\mathrm{P}_{34}$ are determined by $[42,70]$

$$
p\left[k-\frac{1}{2}\right]=k\left(2 \Phi^{2}+\xi\right)-2 \frac{d^{2}}{d \xi^{2}} \ln \psi_{k-1} .
$$

If one proceeds with the case $b=0$ in (4.11), i.e.

$$
\phi(\xi)=\operatorname{Ai}\left(-2^{-1 / 3} \xi\right)
$$

where we have set $a=1$, without loss of generality, then it was established in $[42,70]$ that, for any $k$, there exists a $\xi_{k}$ such that $p\left[k-\frac{1}{2}\right]$ is non-singular and positive on $\xi<\xi_{k}$. This positivity property is depicted graphically for $k=1,2, \ldots, 6$ in [70].

\section{Conclusion}

The aim of the present work has been to exploit an integrable Ermakov-Painlevé II reduction of a resonant NLS encapsulation of a nonlinear cold plasma system to generate classes of similarity solutions in terms of either Yablonskii-Vorob'ev polynomials or classical Airy functions. It is remarked that classes of similarity solutions to the related $\mathrm{P}_{\mathrm{II}}$ equation have recently been shown to provide exact solutions to nonlinear moving boundary problems for certain solitonic equations [73-75] while similarity solutions in magneto-gasdynamics descriptive of plasma columns confined by moving boundaries have been isolated in [22].

\section{References}

[1] H. Washimi and T. Taniuti, Propagation of ion-acoustic solitary waves of small amplitude, Phys. Rev. Lett. 17, 996-998 (1966).

[2] Y.A. Berezin and V.I. Karpman, Theory of non-stationary finite amplitude waves in low density plasma, Soviet Phys. JETP 19, 1265-1271 (1974).

[3] Y.A. Berezin and V.I. Karpman, Nonlinear evolution of disturbances in plasmas and other dispersive media, Soviet Phys. JETP 24, 1049-1056 (1967).

[4] A. Jeffrey, The role of the Korteweg-de Vries equation in plasma physics, Q. J. L. Roy. Astron. Soc. 14, 183-189 (1973). 
[5] T. Kakutani, H. Ono, T. Taniuti and C.C. Wei, Reduction perturbation method in nonlinear wave propagation II: application to hydromagnetic waves in a cold plasma, J. Phys. Soc. Japan 24, 11591166 (1968).

[6] T. Kakutani and H. Ono, Weak nonlinear hydromagnetic waves in cold collisionless plasma, J. Phys. Soc. Japan 26, 1305-1318 (1969).

[7] B.D. Fried and Y.H. Ichikawa, On the nonlinear Schrödinger equation for Langmuir waves, J. Phys. Soc. Japan 34, 1073-1082 (1973).

[8] Y.H. Ichikawa, T. Imamura and T. Taniuti, Nonlinear wave modulation in collisionless plasma, J. Phys. Soc. Japan 33, 189-197 (1972).

[9] K. Shimizu and Y.H. Ichikawa, Automodulation of ion oscillation modes in plasma, J. Phys. Soc. Japan 33, 789-792 (1972).

[10] J. Weiland and H. Wilhelmsson, Coherent Nonlinear Interaction of Waves in Plasmas (International Series in Natural Philosophy, vol. 88), Pergamon, New York and Oxford (1997).

[11] C. Rogers, On the Heisenberg spin equation in hydrodynamics (Informes de Matématics, Série B, vol.127) Instituto de Matématica Pura e Applicada, Rio de Janeriro, Brazil (2000).

[12] C. Rogers and W.K. Schief, On geodesic hydrodynamic motions. Heisenberg spin connections, $J$. MatH. Anal. Appl. 251, 855-870 (2000).

[13] C. Rogers and J.G. Kingston, Non-dissipative magnetohydrodynamic flows with magnetic and velocity field lines orthogonal geodesics on a normal congruence, SIAM J. Applied Math. 26, 183-195 (1974).

[14] C. Rogers and A. Szereszewski, On the geometry of complex-lamellar magnetohydrodynamics. Universal motions, Stud. Appl. Math. 26, 183-195 (2012).

[15] C. Rogers and W.K. Schief, Intrinsic geometry of the NLS equation and its auto-Bäcklund transformation, Stud. Appl. Math. 101, 267-287 (1998).

[16] C. Rogers and W.K. Schief, Bäcklund and Darboux Transformations. Geometry and Modern Applications in Soliton Theory (Cambridge Texts in Applied Mathematics), Cambridge University Press, Cambridge (2002).

[17] W.K. Schief, Hidden integrability in ideal magnetohydrodynamics. The Pohlmeyer-Lund-Regge model, Phys. Plasmas 10, 2677-2685 (2003).

[18] C. Rogers and W.K. Schief, Novel integrable reductions in nonlinear continuum mechanics via geometric constraints, J. Math. Phys. 44, 3341-3369 (2003).

[19] C. Rogers and W.K. Schief, Vortex trains in super-Alfvénic magnetogasdynamics. Application of reciprocal-Bäcklund transformations, J. Nonlinear Math. Phys. 12, Supplement 1, 548-564 (2005).

[20] C. Rogers, J.G. Kingston and W.F. Shadwick, On reciprocal-type invariant transformations in magnetogasdynamics, J. Math. Phys. 21, 395-397 (1980).

[21] C. Rogers, A Ermakov-Ray-Reid reduction in $2+$ 1-dimensional transverse magnetogasdynamics, in: Group analysis of differential equations and integrable systems, Department of Mathematics and Statistics, University of Cyprus, Nicosia (2011) 164-177.

[22] C. Rogers and W.K. Schief, The pulsrodon in $2+$ 1-dimensional magnetogasdynamics. Hamiltonian structure and integrability, J. Math. Phys. 52, 083701 (2011).

[23] C. Rogers, B. Malomed, K. Chow and H. An, Ermakov-Ray-Reid systems in nonlinear optics, J. Phys. A Mathematical \&5 Theoretical 43, 455214 (2010).

[24] C. Rogers, B. Malomed and H. An, Ermakov-Ray-Reid reductions of variational approximations in nonlinear optics, Stud. Appl. Math. 129, 389-413 (2012).

[25] C. Rogers and H. An, Ermakov-Ray-Reid systems in 2+1-dimensional rotating shallow water theory, Stud. Appl. Math. 125, 275-299 (2010).

[26] C. Rogers and H. An, A non-isothermal spinning magnetogasdynamics cloud system. Integrable and Hamiltonian-Ermakov structure, Note di Matematica 32, 175-191 (2012).

[27] F.J. Dyson, Dynamics of a spinning gas cloud, J. Math. Mech. 18, 91-101 (1968).

[28] J.A. Giannini and R.I. Joseph, The role of the second Painlevé transcendent in nonlinear optics, Phys. Lett. A 141, 417-419 (1989). 
[29] A.I. Akhiezer, I.A. Akhiezer, R.V. Polovin, A.G. Sitenko and K.N. Stepanov, Plasma Electrodynamics. Volume 1: Linear theory. Volume 2: Non-linear theory and fluctuations (International Series of Monographs in Natural Philosophy, vols. 68, 80) Pergamon Press, Oxford (1975).

[30] V.I. Karpman, Nonlinear Waves in Dispersive Media, Pergamon, Oxford (1975).

[31] J.H. Lee, O.K. Pashaev, C. Rogers and W.K. Schief, The resonant nonlinear Schrödinger equation in cold plasma physics. Application of Bäcklund-Darboux transformations and superposition principles, J. Plasma Phys. 73, 257-272 (2007).

[32] G.B. Whitham, Nonlinear dispersive waves, Proc. Roy. Soc. London Ser. A 283, 238-261 (1965).

[33] E. Madelung, Quantentheorie in Hydrodynamischen form, Zeit. für Phys. 40, 322-326 (1926).

[34] O.K. Pashaev and J.H. Lee, Resonance solitons as black holes in Madelung fluid, Mod. Phys. Lett. A 17, 1601-1619 (2002).

[35] O.K. Pashaev, J.H. Lee and C. Rogers, Soliton resonances in a generalised nonlinear Schrödinger equation, J. Phys. A: Math. Theor. 41, 452001-452009 (2008).

[36] C. Rogers, Integrable substructure in a Korteweg capillarity model. A Kármán-Tsien type constitutive relation, J. Nonlinear Math. Phys. 21, 74-88 (2014).

[37] M.J. Ablowitz, D.J. Kaup, A.C. Newell and H. Segur, Nonlinear evolution equations of physical significance, Phys. Rev. Lett. 31, 125-127 (1973).

[38] M.J. Ablowitz and P.A. Clarkson, Solitons, Nonlinear Evolution Equations and Inverse Scattering (LMS Lecture Notes on Mathematics, vol. 149), Cambridge University Press, Cambridge (1991).

[39] C. Rogers, A novel Ermakov-Painlevé II system. N+1-dimensional coupled NLS and elastodynamic reductions, Stud. Appl. Math. 133, 2144-231 (2014).

[40] P. Amster and C. Rogers, On a Ermakov-Painlevé II reduction in three-ion electrodiffusion. A Dirichlet boundary value problem, Discrete and Continuous Dynamical Systems 35, 3277-3292 (2015).

[41] V.I. Gromak, Bäcklund transformations of Painlevé equations and their applications, in: The Painlevé Property. One Century Later (CRM Ser. Math. Phys., R. Conte, Editor), Springer-Verlag, New York (1999) 687-734.

[42] L.K. Bass, J.J.C. Nimmo, C. Rogers and W.K. Schief, Electrical structures of interfaces. A Painlevé II model, Proc. Roy. Soc. London Ser. A 466, 2117-2136 (2010).

[43] B. Gambier, Sur les équations différentielles du second ordre et du premier degré dont l'intégrale générale est à points critiques fixés, Acta Math. 33, 1-55 (1910).

[44] N.A. Lukashevich, The second Painlevé equation, Diff. Eq. 7, 853-854 (1971).

[45] V.I. Gromak, I. Laine and S. Shimomura, Painlevé Differential Equations in the Complex Plane (Studies in Mathematics, vol. 28), de Gruyter, Berlin (2002).

[46] P.A. Clarkson, Painlevé equations - nonlinear special functions, in: Orthogonal Polynomials and Special Functions: Computation and Application (Lecture Notes in Math., vol. 1883, F. Màrcellan and W. Van Assche, Editors), Springer-Verlag, Berlin (2006) 331-411.

[47] C. Rogers and W.F. Shadwick, Bäcklund Transformations and Their Applications, Academic Press, New York (1982).

[48] C. Loewner, A transformation theory of partial differential equations of gasdynamics, NACA Technical Note 2065, 1-56 (1950).

[49] C. Loewner, Generation of solutions of systems of partial differential equations by composition of infinitesimal Bäcklund transformations, J. Anal. Math. 2, 219-242 (1952).

[50] A. Seeger, H. Donth and A. Kochendörfer, Theorie der Versetzungen in eindimensionalen Atomreihen III Versetzungen, Eigenbewegungen und ihr Wechselwirkung, Zeit. Phys. 134, 173-193 (1953).

[51] G.L. Lamb Jr, Analytic descriptions of ultrashort optical pulse propagation in a resonant medium, Rev. Mod. Phys. 43, 99-124 (1971).

[52] B.G. Konopelchenko and C. Rogers, On $2+1$-dimensional nonlinear systems of Loewner-type, Phys. Lett. A 158, 391-397 (1991).

[53] B.G. Konopelchenko and C. Rogers, On generalized Loewner systems: novel integrable equations in 2+1-dimensions, J. Math. Phys. 34, 214-242 (1993). 
[54] B.G. Konopelchenko, W.K. Schief and C. Rogers, The 2+1-dimensional sine Gordon system: its auto Bäcklund transformation, Phys. Lett. A 172, 39-46 (1992).

[55] J.J.C. Nimmo, A class of solutions of the Konopelchenko-Rogers equations, Phys. Lett. A 168, 113-119 (1992).

[56] F. Calogero, Universal integrable nonlinear PDEs, in: Application of Analytic and Geometric Methods to Nonlinear Differential Equations (NATO Adv. Sci. Inst. Ser. C Math. Phys. Sci., vol. 413, P.A. Clarkson, Editor), Kluwer, Dordrecht (1993) 109-114.

[57] P.A. Clarkson, E.L. Mansfield and A.E. Milne, Symmetries and exact solutions of a $(2+1)$-dimensional sine-Gordon system, Philos. Trans. Roy. Soc. London Ser. A 354, 1807-1835 (1996).

[58] W.K. Schief, On a $2+1$-dimensional integrable Ernst-type equation, Proc. Roy. Soc. London Ser. A 466, 381-398 (1994).

[59] J.J.C. Nimmo and W.K. Schief, Superposition principles associated with the Moutard transformation: an integrable discretisation of a 2+1-dimensional sine-Gordon system, Proc. Roy. Soc. London Ser. A 453, 255-279 (1997).

[60] W.K. Schief, On The geometry of an integrable 2+1-dimensional sine-Gordon system, Proc. Roy. Soc. London Ser. A 453, 1671-1688 (1997).

[61] S.Y. Lou, C. Rogers and W.K. Schief, Virasoro structure and localised excitations of the LKR system, J. Math. Phys. 44, 5869-5887 (2003).

[62] A.P. Bassom, P.A. Clarkson and A.C. Hicks Bäcklund transformations and solution hierarchies for the fourth Painlevé equation, Stud. Appl. Math. 95, 1-71 (1995).

[63] A.E. Milne, P.A. Clarkson and A.P. Bassom, Bäcklund transformations and solution hierarchies for the third Painlevé equation, Stud. Appl. Math. 98, 139-194 (1997).

[64] A.S. Fokas and Y.C. Yortsos, The transformation properties of the sixth Painlevé equation and oneparameter families of solutions, Lett. Nuovo Cimento 30, 539-544 (1981).

[65] M. Jimbo and T. Miwa, Monodromy preserving deformations of linear ordinary differential equations with rational coefficients. II, Physica D2, 407-448 (1981).

[66] K. Okamoto, Studies on the Painlevé equations III. Second and fourth Painlevé equations, $\mathrm{P}_{\text {II }}$ and $\mathrm{P}_{\text {IV }}$, Math. Ann. 275, 221-255 (1986).

[67] P.J. Forrester and N.S. Witte, Application of the $\tau$-function theory of Painlevé equations to random matrices: PIV, PII and the GUE, Commun. Math. Phys. 219, 357-398 (2001).

[68] A.I. Yablonskii, On rational solutions of the second Painlevé equation, Vesti. Akad. Nauk. BSSR Ser. Fiz. Tkh. Nauk. 3, 30-35 (1959).

[69] A.P. Vorob'ev, On rational solutions of the second Painlevé equation, Diff. Eq. 1, 79-61 (1965).

[70] P.A. Clarkson, On Airy solutions of the second Painlevé equation, Stud. Appl. Math. 137, 93-109 (2016).

[71] P.A. Clarkson, Remarks on the Yablonskii-Vorob'ev polynomials, Phys. Lett. A 319, 137-144 (2003).

[72] P.A. Clarkson and E.L. Mansfield, The second Painlevé equation, its hierarchy and associated special polynomials, Nonlinearity 16, R1-R26 (2003).

[73] C. Rogers, Moving boundary problems for the Harry Dym equation and its reciprocal associates, Zeit. Angew. Math. Phys. 66, 2069-2079 (2015).

[74] C. Rogers, On a class of moving boundary problems for the potential mkdV equation, Special Issue on Waves and Stability, Ricerche di Matematica 65, 563-577 (2016).

[75] C. Rogers, Moving boundary problems for an extended Dym equation. Reciprocal connections, Meccanica 52, 3531-3540 (2017). 